\title{
DEXAMETHASONE - INTRATHECAL MINIMISER OF SIMPLE HAEMATHOLOGIC STRESS BIOMARKERS IN HIP FRACTURE
}

\author{
Livija Šakić ${ }^{1}$, Dinko Tonković ${ }^{2}$ and Kata Šakić ${ }^{3}$ \\ ${ }^{1}$ Department of Anaesthesiology, Reanimatology and Intensive Medicine, \\ University Hospital "Sveti Duh", Zagreb, Croatia; \\ ${ }^{2}$ Department of Anaesthesiology, Reanimatology and Intensive Therapy, \\ University Hospital Centre Zagreb “Rebro”, School of Medicine, University of Zagreb, Zagreb, Croatia; \\ ${ }^{3}$ Department of Anaesthesiology and Pain Therapy at Maxillofacial, General and Plastic surgery \\ Polyclinic "Bagatin", Zagreb, Croatia
}

\begin{abstract}
SUMMARY - Proximal femoral fractures are the most common cause of emergency admission to hospital with high postoperative morbidity. The hypothesis was that a single shot of intrathecal dexamethasone and levobupivacaine in anaesthesia for surgical correction of proximal femoral fracture in elderly patients reduces surgical stress with better quality hospitalisation.

The study included sixty elderly patients with proximal femoral fracture, ASA status 2 and 3, randomised into two groups. The study group of thirty patients received $8 \mathrm{mg}$ of dexamethasone and 12,5 $\mathrm{mg}$ of levobupivacaine $0,5 \%$ intrathecally, DLSA group, and the parallel group of thirty patients received $12,5 \mathrm{mg}$ of levobupivacaine $0,5 \%$ intrathecally, LSA group. Plasma cortisol and glucose concentrations were assessed before and after anaesthesia, pain intensity was evaluated using Visual Analogue Scale score and hospitalisation was analysed. Results showed decreased cortisol concentrations, longer analgesia duration and shorter hospitalisation in the DLSA group. Glucose concentrations did not differ significantly between the patients in either group. Enlightening the study results collected, single shot of intrathecal administration of dexamethasone in spinal anaesthesia for surgical treatment of proximal femoral fractures reduces the stress response by decreasing plasma cortisol concentrations prolonging analgesia with better rehabilitation possibilities, hence shortening hospitalisation which explains this pattern of anaesthesia.
\end{abstract}

Key words: cortisol, glucose, stress, pain, spinal anaesthesia, femoral fracture, hospitalization

\section{Introduction}

There are many definitions of stress by numerous stress researchers. One of the definitions is the one given by the founder of the field of stress research, Seyle, who observed that stress is produced by psychological threat and insult to the body-self as well as by physical injury and disease $\mathrm{e}^{1,2}$. The disruption of homeostasis by psychological and physical stress activates

Correspondence to: Livija Šakic, $M D, P h D$, Department of Anaesthesiology, Reanimatology and Intensive Medicine, University Hospital "Sveti Duh", Zagreb, Croatia

e-mail: mitzilivilla@yahoo.com programmes of neural, hormonal, immunological and behavioural activity selected from a genetically determined repertoire ${ }^{3,4}$. The glucocorticoid cortisol is an essential hormone for survival because it is responsible for producing and maintaining high levels of glucose necessary for the response to stress. However, it is potentially destructive, because it breaks down the protein in muscles and inhibits the ongoing replacement of calcium in bones, in order to ensure a high glucose level ${ }^{2}$.

Perioperative pain in elderly patients with proximal femoral fractures is an utmost stress trigger where injury and surgical treatment strongly stimulate the ad- 
renocorticotrophic $(\mathrm{ACTH})$ hormone release which then stimulates adrenal secretion of glucocorticoids with substantially high cortisol concentrations in plasma. The initial metabolic response to perioperative tissue damage involves greater availability of glucose, amino acids, and free fatty acids and coincides with an activation of the hypothalamic-pituitary-adrenal (HPA) axis ${ }^{5}$. These patients are the most vulnerable population to the deleterious effects of poorly managed pain, adverse effects of both drugs and postoperative pain. Therefore, achieving effective analgesia is particularly difficult because it is necessary to personalise the treatments and, at the same time, the ineffective analgesia may lead to serious complications ${ }^{6}$. Postoperative hyperglycaemia may lead to infection of surgical wounds and prolong the hospitalisation? Dexamethasone, a synthetic glucocorticoid, with antiimflammatory and immunosuppressive characteristics can diffuse into the central nervous system (CNS). Dexamethasone inhibits the HPA axis primarily on the pituitary level by transcription mechanisms in the cells, but not obligatory for ACTH gene synthesis ${ }^{8}$. It is comprehended that a harmful stimulus can suppress the HPA axis feedback information, which is a possible function of nature and intensity of the stimulus.

The aim of this study was to establish the changes of cortisol and glucose plasma concentrations by a single shot of intrathecal dexamethasone and levobupivacaine in anaesthesia for surgical correction of proximal femoral fracture and its effect on analgesia and hospitalisation.

\section{Methods}

This clinical study was conducted from 2013-2016 at the University Hospital "Sveti Duh", Zagreb. Hospital's ethics committee approval was obtained and the patients read and signed the informed consent after the study has been explained to them.

Sixty patients with proximal femoral fracture, American society of anesthesiologists (ASA) physical status 2 or 3, male or female, admitted through hospital's Emergency Department were included in this randomised, prospective, clinical study. All patients with proximal femoral fracture were randomised into two groups after a regular anaesthesiological assessment and by www.randomizer.org. After the final anaesthesiological decision every patient underwent surgical procedure in spinal anaesthesia.

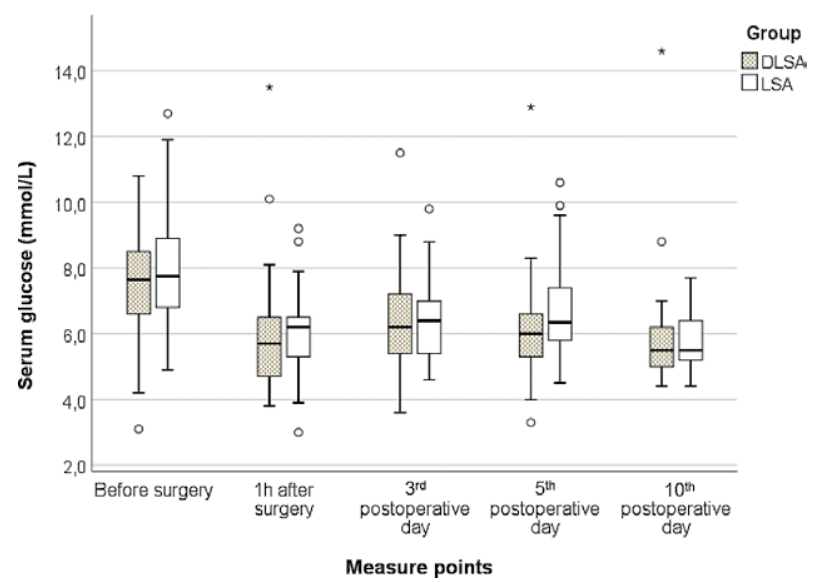

Figure 1: Graphical presentation of serum glucose concentrations between the groups in the study.

DLSA group that received dexamethasone + levobupivacaine spinal anaesthesia; LSA group that received levobupivacaine spinal anaesthesia

Inclusion criteria covered all cooperative elderly patients with proximal femoral fractures of one leg ASA grade 2 or 3 willing to participate in the study. Exclusion criteria were pre-existing cognitive impairment, diabetes mellitus, neurological conditions or tumours, neuroendocrine disorders or tumours, ASA $\geq 4$ and unwillingness to participate in the study or breaking the study protocol. Patients no longer wanting to participate in the study or when unexpected events happened after the study already started were also considered exclusion criteria.

Spinal anaesthesia was performed in the sitting position on intervertebral levels L2-L3 or L3-L4 with middle line approach using BD Spinal Needle Quincke Type Point 22-27 GA.

Thirty patients in the study group received $8 \mathrm{mg}$ of dexamethasone (Dexamethasone Sodium Phosphate, KRKA) and $12.5 \mathrm{mg}$ of $0.5 \%$ levobupivacaine (Chirochaine, Abbott) administered intrathecally, the DLSA group. The control group of thirty patients received $12.5 \mathrm{mg}$ of $0.5 \%$ levobupivacaine intrathecally, the LSA group.

The primary outcomes were to define if intrathecal dexamethasone effects perioperative plasma cortisol and glucose fluctuations. Plasma cortisol was assessed in five measure points: T1- before anaesthesia and infusion administration, T2- one hour after surgery, T3- third postoperative day at $6 \mathrm{am}, \mathrm{T} 4$ - fifth postoperative day at 6 am and T5- on tenth postoperative day 
Table 1. Spearman's correlation analysis in the DLSA group: Correlations of clinical parameters with VAS scores, cortisol levels and glucose concentrations before and after surgery.

\begin{tabular}{|c|c|c|c|c|c|c|}
\hline & & $\begin{array}{l}\text { Postoperative } \\
\text { length of hospital } \\
\text { stay (days) }\end{array}$ & $\begin{array}{l}\text { Preoperative } \\
\text { length of hospital } \\
\text { stay (days) }\end{array}$ & $\begin{array}{l}\text { Duration } \\
\text { of surgery } \\
\text { (min) }\end{array}$ & $\begin{array}{l}\text { Analgesia } \\
\text { duration } \\
(\mathrm{min})\end{array}$ & $\begin{array}{l}\text { Hospitalisation } \\
\text { (days) }\end{array}$ \\
\hline VAS preoperatively & $\begin{array}{l}\text { rho } \\
\mathrm{P}\end{array}$ & $\begin{array}{l}-0.175 \\
0.355\end{array}$ & $\begin{array}{l}-0.248 \\
0.186\end{array}$ & $\begin{array}{l}0.216 \\
0.252\end{array}$ & $\begin{array}{l}0.080 \\
0.675\end{array}$ & $\begin{array}{l}-0.314 \\
0.091\end{array}$ \\
\hline $\begin{array}{l}\text { VAS } 1 \text { hour } \\
\text { postoperatively }\end{array}$ & $\begin{array}{l}\text { rho } \\
\mathrm{P}\end{array}$ & NA & NA & NA & NA & NA \\
\hline $\begin{array}{l}\text { VAS } 3^{\text {rd day }} \\
\text { postoperatively }\end{array}$ & $\begin{array}{l}\text { rho } \\
\mathrm{P}\end{array}$ & $\begin{array}{l}-0.331 \\
0.074\end{array}$ & $\begin{array}{l}0.112 \\
0.557\end{array}$ & $\begin{array}{l}0.148 \\
0.436\end{array}$ & $\begin{array}{l}-0.005 \\
0.980\end{array}$ & $\begin{array}{l}0.020 \\
0.917\end{array}$ \\
\hline $\begin{array}{l}\text { VAS } 5^{\text {th }} \text { day } \\
\text { postoperatively }\end{array}$ & $\begin{array}{l}\text { rho } \\
\mathrm{P}\end{array}$ & $\begin{array}{l}-0.182 \\
0.336\end{array}$ & \begin{tabular}{|l|}
-0.188 \\
0.321 \\
\end{tabular} & $\begin{array}{l}0.154 \\
0.415\end{array}$ & \begin{tabular}{|l|}
-0.289 \\
0.121 \\
\end{tabular} & $\begin{array}{l}-0.150 \\
0.428\end{array}$ \\
\hline $\begin{array}{l}\text { VAS } 10^{\text {th }} \text { day } \\
\text { postoperatively }\end{array}$ & $\begin{array}{l}\text { rho } \\
\mathrm{P}\end{array}$ & $\begin{array}{l}-0.357 \\
0.053\end{array}$ & $\begin{array}{l}-0.061 \\
0.751 \\
\end{array}$ & $\begin{array}{l}0.126 \\
0.508\end{array}$ & $\begin{array}{l}-0.347 \\
0.061 \\
\end{array}$ & $\begin{array}{l}-0.181 \\
0.338\end{array}$ \\
\hline $\begin{array}{l}\text { Cortisol } \\
\text { preoperatively }\end{array}$ & $\begin{array}{l}\text { rho } \\
\text { P }\end{array}$ & $\begin{array}{l}-0.083 \\
0.664\end{array}$ & \begin{tabular}{|l|}
-0.439 \\
$\mathbf{0 . 0 1 5}$
\end{tabular} & $\begin{array}{l}0.048 \\
0.801\end{array}$ & $\begin{array}{l}0.187 \\
0.322\end{array}$ & $\begin{array}{l}-0.348 \\
0.059\end{array}$ \\
\hline $\begin{array}{l}\text { Cortisol } 1 \text { hour } \\
\text { postoperatively }\end{array}$ & $\begin{array}{l}\text { rho } \\
\mathrm{P}\end{array}$ & $\begin{array}{l}-0.191 \\
0.312\end{array}$ & \begin{tabular}{|l|}
-0.467 \\
$\mathbf{0 . 0 0 9}$
\end{tabular} & $\begin{array}{l}-0.021 \\
0.913 \\
\end{array}$ & $\begin{array}{l}-0.031 \\
0.871\end{array}$ & $\begin{array}{l}-0.417 \\
\mathbf{0 . 0 2 2}\end{array}$ \\
\hline $\begin{array}{l}\text { Cortisol } 3^{\text {rd day }} \\
\text { postoperatively }\end{array}$ & $\begin{array}{l}\text { rho } \\
\mathrm{P}\end{array}$ & $\begin{array}{l}-0.111 \\
0.559\end{array}$ & $\begin{array}{l}-0.170 \\
0.368\end{array}$ & $\begin{array}{l}0.327 \\
0.077\end{array}$ & \begin{tabular}{|l|}
-0.236 \\
0.209
\end{tabular} & $\begin{array}{l}-0.284 \\
0.128\end{array}$ \\
\hline $\begin{array}{l}\text { Cortisol } 5^{\text {th }} \text { day } \\
\text { postoperatively }\end{array}$ & $\begin{array}{l}\text { rho } \\
\mathrm{P}\end{array}$ & $\begin{array}{l}0.320 \\
0.084\end{array}$ & $\begin{array}{l}0.016 \\
0.933\end{array}$ & $\begin{array}{l}-0.093 \\
0.624\end{array}$ & $\begin{array}{l}-0.006 \\
0.976\end{array}$ & $\begin{array}{l}0.141 \\
0.459\end{array}$ \\
\hline $\begin{array}{l}\text { Cortisol } 10^{\text {th }} \text { day } \\
\text { postoperatively }\end{array}$ & $\begin{array}{l}\text { rho } \\
\text { P }\end{array}$ & $\begin{array}{l}0.224 \\
0.235\end{array}$ & $\begin{array}{l}-0.021 \\
0.914 \\
\end{array}$ & $\begin{array}{l}-0.129 \\
0.496 \\
\end{array}$ & $\begin{array}{l}0.031 \\
0.870\end{array}$ & $\begin{array}{l}0.126 \\
0.506\end{array}$ \\
\hline $\begin{array}{l}\text { Serum glucose } \\
\text { preoperatively }\end{array}$ & $\begin{array}{l}\text { rho } \\
\mathrm{P}\end{array}$ & $\begin{array}{l}0.108 \\
0.569\end{array}$ & $\begin{array}{l}0.135 \\
0.477\end{array}$ & $\begin{array}{l}-0.018 \\
0.926\end{array}$ & $\begin{array}{l}0.009 \\
0.962\end{array}$ & $\begin{array}{l}0.203 \\
0.283\end{array}$ \\
\hline $\begin{array}{l}\text { Serum } 1 \text { hour } \\
\text { postoperatively }\end{array}$ & $\begin{array}{l}\text { rho } \\
\mathrm{P}\end{array}$ & $\begin{array}{l}-0.135 \\
0.478\end{array}$ & $\begin{array}{l}-0.359 \\
0.051\end{array}$ & $\begin{array}{l}0.145 \\
0.445\end{array}$ & $\begin{array}{l}-0.243 \\
0.195\end{array}$ & $\begin{array}{l}-0.340 \\
0.066\end{array}$ \\
\hline $\begin{array}{l}\text { Serum } 3^{\text {rd }} \text { day } \\
\text { postoperatively }\end{array}$ & $\begin{array}{l}\text { rho } \\
\mathrm{P}\end{array}$ & $\begin{array}{l}-0.226 \\
0.229\end{array}$ & $\begin{array}{l}-0.480 \\
0.007\end{array}$ & $\begin{array}{l}0.230 \\
0.221\end{array}$ & \begin{tabular}{|l|}
-0.038 \\
0.841 \\
\end{tabular} & \begin{tabular}{|l|}
-0.519 \\
$\mathbf{0 . 0 0 3}$
\end{tabular} \\
\hline $\begin{array}{l}\text { Serum } 5^{\text {th }} \text { day } \\
\text { postoperatively }\end{array}$ & $\begin{array}{l}\text { rho } \\
\mathrm{P}\end{array}$ & $\begin{array}{l}-0.040 \\
0.833 \\
\end{array}$ & $\begin{array}{l}-0.160 \\
0.400 \\
\end{array}$ & $\begin{array}{l}0.033 \\
0.863\end{array}$ & $\begin{array}{l}0.013 \\
0.944\end{array}$ & $\begin{array}{l}-0.227 \\
0.228 \\
\end{array}$ \\
\hline $\begin{array}{l}\text { Serum } 10^{\text {th }} \text { day } \\
\text { postoperatively }\end{array}$ & $\begin{array}{l}\text { rho } \\
\mathrm{P}\end{array}$ & $\begin{array}{l}-0.152 \\
0.424 \\
\end{array}$ & \begin{tabular}{|l|}
-0.411 \\
$\mathbf{0 . 0 2 4}$ \\
\end{tabular} & $\begin{array}{l}0.016 \\
0.932 \\
\end{array}$ & $\begin{array}{l}0.099 \\
0.602 \\
\end{array}$ & $\begin{array}{l}-0.409 \\
\mathbf{0 . 0 2 5}\end{array}$ \\
\hline
\end{tabular}

DLSA group that received dexamethasone + levobupivacaine spinal anaesthesia

at 6 am 0.5 millilitres $(\mathrm{ml})$ of patients' blood was taken for single measurement. Cortisol was assayed by Roche Elecsys Immunoassay System in laboratory reference points (185-624) nmol/L. Serum glucose was assessed in five measure points at the same time as cortisol was measured. $0.2 \mathrm{ml}$ of patients' blood was taken for single measurement. Glucose was assayed by Beckman Coulter AU 400 and AU 680 apparatus or Olympus
Analyzer 2 in laboratory reference points (4.4-6.4) $\mathrm{mmol} / \mathrm{L}$.

As secondary outcomes we evaluated howintrathecal dexamethasone with levobupivacaine anaesthetic pattern was affecting quality of analgesia and length of hospital stay. Visual Analogue Scale (VAS), in scores from 0 - 10, was used for evaluating pain intensity every three hours when patients were 
Table 2. Spearman's correlation analysis in the LSA group: Correlations of clinical parameters with VAS scores, cortisol levels and glucose concentrations before and after surgery.

\begin{tabular}{|c|c|c|c|c|c|c|}
\hline & & $\begin{array}{l}\text { Postoperative } \\
\text { length of hospital } \\
\text { stay (days) }\end{array}$ & $\begin{array}{l}\text { Preoperative } \\
\text { length of hospital } \\
\text { stay (days) }\end{array}$ & \begin{tabular}{|l}
$\begin{array}{l}\text { Duration } \\
\text { of surgery } \\
(\mathrm{min})\end{array}$ \\
\end{tabular} & $\begin{array}{l}\text { Analgesia } \\
\text { duration } \\
(\mathrm{min}) \\
\end{array}$ & $\begin{array}{l}\text { Hospitalisation } \\
\text { (days) }\end{array}$ \\
\hline VAS preoperatively & $\begin{array}{l}\text { rho } \\
\text { P }\end{array}$ & $\begin{array}{l}-0.127 \\
0.503\end{array}$ & $\begin{array}{l}-0.114 \\
0.550 \\
\end{array}$ & $\begin{array}{l}-0.035 \\
0.856 \\
\end{array}$ & $\begin{array}{l}-0.334 \\
0.071\end{array}$ & $\begin{array}{l}0.043 \\
0.823\end{array}$ \\
\hline $\begin{array}{l}\text { VAS } 1 \text { hour } \\
\text { postoperatively }\end{array}$ & $\begin{array}{l}\text { rho } \\
\mathrm{P}\end{array}$ & $\begin{array}{l}-0.039 \\
0.838\end{array}$ & $\begin{array}{l}0.261 \\
0.164\end{array}$ & $\begin{array}{l}0.308 \\
0.098\end{array}$ & $\begin{array}{l}-0.487 \\
0.006\end{array}$ & $\begin{array}{l}0.154 \\
0.416\end{array}$ \\
\hline $\begin{array}{l}\text { VAS } 3^{\text {rd }} \text { day } \\
\text { postoperatively }\end{array}$ & $\begin{array}{l}\text { rho } \\
\mathrm{P}\end{array}$ & \begin{tabular}{|l|}
-0.270 \\
0.149
\end{tabular} & $\begin{array}{l}-0.231 \\
0.220\end{array}$ & $\begin{array}{l}0.174 \\
0.359\end{array}$ & $\begin{array}{l}-0.349 \\
0.059\end{array}$ & $\begin{array}{l}-0.300 \\
0.107\end{array}$ \\
\hline $\begin{array}{l}\text { VAS } 5^{\text {th }} \text { day } \\
\text { postoperatively }\end{array}$ & $\begin{array}{l}\text { rho } \\
\text { P }\end{array}$ & $\begin{array}{l}-0.174 \\
0.357\end{array}$ & $\begin{array}{l}-0.051 \\
0.789\end{array}$ & $\begin{array}{l}-0.045 \\
0.812 \\
\end{array}$ & $\begin{array}{l}-0.451 \\
\mathbf{0 . 0 1 2}\end{array}$ & $\begin{array}{l}-0.221 \\
0.241 \\
\end{array}$ \\
\hline $\begin{array}{l}\text { VAS } 10^{\text {th }} \text { day } \\
\text { postoperatively }\end{array}$ & $\begin{array}{l}\text { rho } \\
\mathrm{P}\end{array}$ & $\begin{array}{l}-0.099 \\
0.603 \\
\end{array}$ & $\begin{array}{l}0.225 \\
0.231\end{array}$ & $\begin{array}{l}0.070 \\
0.712 \\
\end{array}$ & $\begin{array}{l}-0.486 \\
\mathbf{0 . 0 0 6} \\
\end{array}$ & $\begin{array}{l}-0.032 \\
0.867 \\
\end{array}$ \\
\hline $\begin{array}{l}\text { Cortisol } \\
\text { preoperatively }\end{array}$ & $\begin{array}{l}\text { rho } \\
\text { P }\end{array}$ & $\begin{array}{l}0.269 \\
0.151\end{array}$ & $\begin{array}{l}0.108 \\
0.569\end{array}$ & $\begin{array}{l}-0.037 \\
0.847\end{array}$ & $\begin{array}{l}-0.267 \\
0.154\end{array}$ & $\begin{array}{l}0.367 \\
\mathbf{0 . 0 4 6}\end{array}$ \\
\hline $\begin{array}{l}\text { Cortisol } 1 \text { hour } \\
\text { postoperatively }\end{array}$ & $\begin{array}{l}\text { rho } \\
\mathrm{P}\end{array}$ & $\begin{array}{l}-0.123 \\
0.519\end{array}$ & $\begin{array}{l}0.091 \\
0.631\end{array}$ & $\begin{array}{l}0.193 \\
0.307\end{array}$ & $\begin{array}{l}-0.325 \\
0.080\end{array}$ & $\begin{array}{l}0.078 \\
0.681\end{array}$ \\
\hline $\begin{array}{l}\text { Cortisol } 3^{\text {rd }} \text { day } \\
\text { postoperatively }\end{array}$ & $\begin{array}{l}\text { rho } \\
\mathrm{P}\end{array}$ & $\begin{array}{l}-0.201 \\
0.286\end{array}$ & $\begin{array}{l}-0.147 \\
0.437 \\
\end{array}$ & $\begin{array}{l}0.004 \\
0.984\end{array}$ & $\begin{array}{l}-0.308 \\
0.097\end{array}$ & $\begin{array}{l}-0.154 \\
0.417\end{array}$ \\
\hline $\begin{array}{l}\text { Cortisol } 5^{\text {th }} \text { day } \\
\text { postoperatively. }\end{array}$ & $\begin{array}{l}\text { rho } \\
\text { P }\end{array}$ & $\begin{array}{l}-0.156 \\
0.411\end{array}$ & $\begin{array}{l}-0.011 \\
0.956\end{array}$ & $\begin{array}{l}-0.238 \\
0.205\end{array}$ & $\begin{array}{l}-0.332 \\
0.073\end{array}$ & $\begin{array}{l}-0.067 \\
0.724\end{array}$ \\
\hline $\begin{array}{l}\text { Cortisol } 10^{\text {th }} \text { day } \\
\text { postoperatively }\end{array}$ & $\begin{array}{l}\text { rho } \\
\text { P }\end{array}$ & $\begin{array}{l}0.031 \\
0.872\end{array}$ & \begin{tabular}{|l|}
-0.097 \\
0.611 \\
\end{tabular} & $\begin{array}{l}0.154 \\
0.415\end{array}$ & $\begin{array}{l}-0.534 \\
\mathbf{0 . 0 0 2}\end{array}$ & $\begin{array}{l}-0.006 \\
0.973\end{array}$ \\
\hline $\begin{array}{l}\text { Serum glucose } \\
\text { preoperatively }\end{array}$ & $\begin{array}{l}\text { rho } \\
\mathrm{P}\end{array}$ & $\begin{array}{l}-0.065 \\
0.731\end{array}$ & $\begin{array}{l}0.263 \\
0.160\end{array}$ & $\begin{array}{l}-0.020 \\
0.917\end{array}$ & $\begin{array}{l}-0.194 \\
0.304\end{array}$ & $\begin{array}{l}0.085 \\
0.656\end{array}$ \\
\hline $\begin{array}{l}\text { Serum glucose } 1 \\
\text { hour postoperatively }\end{array}$ & $\begin{array}{l}\text { rho } \\
\mathrm{P}\end{array}$ & $\begin{array}{l}0.071 \\
0.710\end{array}$ & $\begin{array}{l}-0.118 \\
0.536 \\
\end{array}$ & $\begin{array}{l}0.389 \\
\mathbf{0 . 0 3 4}\end{array}$ & $\begin{array}{l}-0.324 \\
0.081\end{array}$ & $\begin{array}{l}-0.025 \\
0.895 \\
\end{array}$ \\
\hline $\begin{array}{l}\text { Serum glucose } 3^{\text {rd }} \\
\text { day postoperatively }\end{array}$ & $\begin{array}{l}\text { rho } \\
\text { P }\end{array}$ & $\begin{array}{l}-0.224 \\
0.235\end{array}$ & $\begin{array}{l}-0.143 \\
0.450 \\
\end{array}$ & $\begin{array}{l}-0.072 \\
0.707 \\
\end{array}$ & $\begin{array}{l}-0.130 \\
0.494\end{array}$ & $\begin{array}{l}-0.267 \\
0.154\end{array}$ \\
\hline $\begin{array}{l}\text { Serum glucose } 5^{\text {th }} \\
\text { day postoperatively }\end{array}$ & $\begin{array}{l}\text { rho } \\
\text { P }\end{array}$ & $\begin{array}{l}0.118 \\
0.534\end{array}$ & $\begin{array}{l}-0.119 \\
0.531 \\
\end{array}$ & $\begin{array}{l}-0.067 \\
0.726 \\
\end{array}$ & $\begin{array}{l}-0.043 \\
0.822\end{array}$ & $\begin{array}{l}0.126 \\
0.505\end{array}$ \\
\hline $\begin{array}{l}\text { Serum glucose } 10^{\text {th }} \\
\text { day postoperatively }\end{array}$ & $\begin{array}{l}\text { rho } \\
\text { P }\end{array}$ & $\begin{array}{l}0.013 \\
0.946 \\
\end{array}$ & \begin{tabular}{|l|}
-0.181 \\
0.339 \\
\end{tabular} & $\begin{array}{l}0.543 \\
0.002 \\
\end{array}$ & $\begin{array}{l}-0.082 \\
0.665 \\
\end{array}$ & \begin{tabular}{|l|}
-0.072 \\
0.705 \\
\end{tabular} \\
\hline
\end{tabular}

LSA group that received levobupivacaine spinal anaesthesia

awake in five measure points: T1- before anaesthesia and infusion administration, T2- one hour after surgery, T3- third postoperative day, T4- fifth postoperative day and T5- on tenth postoperative day to follow cortisol measurements. Hospitalisation was calculated by the number of days preoperatively from the day of admission to the day of surgical treatment and postoperatively from the day of surgical reconstruction to the day of hospital discharge in the time period of up to 30 days.

The statistics data are shown in tables and figures, variables that were normally distributed were tested by Kolmogorov-Smirnov test. Categorical variables were shown by using absolute frequencies and ratios, quantitative variables were expressed as median scores and interquartile ranges. The data for descriptive variables 
Table 3. Differences between the groups in VAS scores: Mann-Whitney U test.

\begin{tabular}{|c|c|c|c|c|c|c|c|c|}
\hline \multirow{2}{*}{\multicolumn{2}{|c|}{ Group }} & \multirow{3}{*}{$\begin{array}{l}\mathrm{N} \\
30 \\
30\end{array}$} & \multirow{3}{*}{$\begin{array}{l}\begin{array}{l}\text { Arithmetic } \\
\text { mean }\end{array} \\
8.4 \\
8.1\end{array}$} & \multirow{3}{*}{\begin{tabular}{|l} 
SD \\
1.2 \\
1.3
\end{tabular}} & \multicolumn{3}{|c|}{ Centile } & \multirow{3}{*}{$\begin{array}{l}\mathrm{P} \\
0.374\end{array}$} \\
\hline & & & & & \multirow{2}{*}{\begin{tabular}{|l|}
25. \\
8.0 \\
7.0
\end{tabular}} & \multirow{2}{*}{$\begin{array}{l}\text { Median } \\
8.5 \\
8.0\end{array}$} & \multirow{2}{*}{\begin{tabular}{|l|}
75. \\
9.0 \\
9.0
\end{tabular}} & \\
\hline $\begin{array}{l}\text { VAS } \\
\text { preoperatively }\end{array}$ & $\begin{array}{l}\text { DLSA } \\
\text { LSA }\end{array}$ & & & & & & & \\
\hline $\begin{array}{l}\text { VAS } 1 \text { hour } \\
\text { postoperatively }\end{array}$ & $\begin{array}{l}\text { DLSA } \\
\text { LSA }\end{array}$ & $\begin{array}{l}30 \\
30\end{array}$ & $\begin{array}{l}0.0 \\
0.5\end{array}$ & $\begin{array}{l}0.0 \\
1.3\end{array}$ & $\begin{array}{l}0.0 \\
0.0\end{array}$ & $\begin{array}{l}0.0 \\
0.0\end{array}$ & $\begin{array}{l}0.0 \\
0.0\end{array}$ & 0.061 \\
\hline $\begin{array}{l}\text { VAS } 3^{\text {rd }} \text { day } \\
\text { postoperatively }\end{array}$ & $\begin{array}{l}\text { DLSA } \\
\text { LSA }\end{array}$ & $\begin{array}{l}30 \\
30\end{array}$ & $\begin{array}{l}1.7 \\
3.0\end{array}$ & $\begin{array}{l}1.4 \\
1.3\end{array}$ & $\begin{array}{l}1.0 \\
2.0\end{array}$ & $\begin{array}{l}1.5 \\
3.0\end{array}$ & $\begin{array}{l}2.0 \\
4.0\end{array}$ & $<0.001$ \\
\hline $\begin{array}{l}\text { VAS } 5^{\text {th }} \text { day } \\
\text { postoperatively }\end{array}$ & $\begin{array}{l}\text { DLSA } \\
\text { LSA }\end{array}$ & $\begin{array}{l}30 \\
30\end{array}$ & $\begin{array}{l}0.9 \\
2.6\end{array}$ & $\begin{array}{l}0.8 \\
1.1\end{array}$ & $\begin{array}{l}0.0 \\
2.0\end{array}$ & $\begin{array}{l}1.0 \\
3.0\end{array}$ & $\begin{array}{l}2.0 \\
3.0\end{array}$ & $<0.001$ \\
\hline $\begin{array}{l}\text { VAS } 10^{\text {th }} \text { day } \\
\text { postoperatively }\end{array}$ & $\begin{array}{l}\text { DLSA } \\
\text { LSA }\end{array}$ & $\begin{array}{l}30 \\
30\end{array}$ & $\begin{array}{l}0.5 \\
1.2\end{array}$ & $\begin{array}{l}0.6 \\
0.9\end{array}$ & $\begin{array}{l}0.0 \\
1.0\end{array}$ & $\begin{array}{l}0.0 \\
1.0\end{array}$ & $\begin{array}{l}1.0 \\
2.0\end{array}$ & 0.002 \\
\hline
\end{tabular}

DLSA group that received dexamethasone + levobupivacaine spinal anaesthesia; LSA group that received levobupivacaine spinal anaesthesia

Table 4. Differences between the groups in the length of hospital stay and analgesia duration.

\begin{tabular}{|c|c|c|c|c|c|c|c|c|}
\hline \multirow{2}{*}{\multicolumn{2}{|c|}{ Group }} & \multirow{2}{*}{$\mathrm{N}$} & \multirow{2}{*}{$\begin{array}{l}\text { Arithmetic } \\
\text { mean }\end{array}$} & \multirow{2}{*}{ SD } & \multicolumn{3}{|l|}{ Centile } & \multirow{2}{*}{$\mathrm{P}$} \\
\hline & & & & & 25. & Median & 75. & \\
\hline \multirow{2}{*}{$\begin{array}{l}\text { Preoperative } \\
\text { hospital stay (days) }\end{array}$} & DLSA & 30 & 5.33 & 5.19 & 2.00 & 3.00 & 6.50 & \multirow{2}{*}{0.166} \\
\hline & LSA & 30 & 5.30 & 2.60 & 3.00 & 5.00 & 7.00 & \\
\hline \multirow{2}{*}{$\begin{array}{l}\text { Duration of } \\
\text { analgesia (minutes) }\end{array}$} & DLSA & 30 & 556.90 & 118.45 & 455.75 & 540.00 & 720.00 & \multirow{2}{*}{$<0.001$} \\
\hline & LSA & 30 & 312.30 & 70.79 & 254.75 & 330.00 & 377.00 & \\
\hline \multirow{2}{*}{$\begin{array}{l}\text { Hospitalisation } \\
\text { (days) }\end{array}$} & DLSA & 30 & 15.90 & 6.00 & 11.00 & 14.50 & 18.50 & \multirow{2}{*}{0.045} \\
\hline & LSA & 30 & 17.40 & 4.00 & 15.00 & 19.00 & 20.00 & \\
\hline \multirow{2}{*}{$\begin{array}{l}\text { Postoperative } \\
\text { hospital stay (days) }\end{array}$} & DLSA & 30 & 10.57 & 2.19 & 9.00 & 11.00 & 12.00 & \multirow{2}{*}{0.044} \\
\hline & LSA & 30 & 12.10 & 3.11 & 10.75 & 11.50 & 13.00 & \\
\hline
\end{tabular}

DLSA group that received dexamethasone + levobupivacaine spinal anaesthesia; LSA group that received levobupivacaine spinal anaesthesia

were analysed by chi-square test, though differences in quantitative variables were tested by Mann-Whitney $\mathrm{U}$ test. Particular clinical parameters were evaluated by Spearman's correlation analysis. $\mathrm{P}<0.05$ was considered to be statistically significant. Statistical analysis was performed by the IBM SPSS Statistics for Windows 23 .

\section{Results}

Sixty patients included in the study with mean age 80.65 (range 52-95) years old of which $90 \%$ of participants were older than 70 years of age with proximal femoral fracture of one leg who underwent surgical fixation in spinal anaesthesia. Precisely, $70 \%$ of patients were ASA status 2, the other $30 \%$ were ASA status 3 .

The study results showed no significant differences in cortisol values before surgery in both groups. Cortisol concentrations one hour after surgery were borderline $(\mathrm{P}=0.088)$, although over-all cortisol values in the DLSA group were decreased at all times during postoperative length of hospital stay within laboratory reference points, discreetly elevated or even slightly below the reference points. Plasma cortisol concentrations in all patients were greater in the control group, 
the LSA group, and persisted high in the control group after surgery as well.

Changes in serum glucose concentrations did not show statistically significant differences between the groups in the study (Fig. 1).

VAS scores in the study group, the DLSA group, had no significant correlations with assessed clinical parameters before and after surgical treatment, while cortisol levels negatively correlated with preoperative hospital stay $(\mathrm{P}=0.015)$, one hour postoperatively $(\mathrm{P}=0.009)$ and hospitalisation $(\mathrm{P}=0.022)$. Serum glucose data negatively correlated with preoperative hospital stay measured on the third postoperative day $(\mathrm{P}=0.007)$ and the tenth postoperative day measurement $(\mathrm{P}=0.024)$ and similarly negatively correlated with over-all length of hospital stay with significant $\mathrm{P}$ values $(\mathrm{P}=0.003)$ on the third postoperative day and $(\mathrm{P}=0.025)$ on the tenth postoperative day (Table 1$)$.

The study results showed negative correlation in VAS scores and duration of analgesia one hour after surgery and on the tenth postoperative day measurement $(\mathrm{P}=0.006)$; cortisol levels positively correlated with hospitalisation $(\mathrm{P}=0.046)$, and negatively with duration of analgesia on the tenth postoperative day $(\mathrm{P}=0.002)$ in the LSA group. Serum glucose statistics data showed positive correlation with the duration of surgery measured one hour after surgery $(\mathrm{P}=0.034)$ and on the tenth postoperative day $(\mathrm{P}=0.002)$ in the same group (Table 2).

Perioperative pain intensity in VAS scores data showed considerable differences between the groups in the study with significantly lower, tolerable VAS scores in the study group evaluated on the third $(\mathrm{P}<0.001)$, fifth $(\mathrm{P}<0.001)$ and tenth postoperative day $(\mathrm{P}=0.002)$ and without opioid analgesic requirements (Table 3 ).

Duration of analgesia was longer in the study group, the DLSA group $(\mathrm{P}<0.001)$, hospitalisation was shorter in the study group $(\mathrm{P}=0.045)$, as well as postoperative length of hospital stay $(\mathrm{P}=0.044)$, in comparison to the control group (Table 4).

Hospitalisation lasted approximately 14.5 days (range 9.0-23.0) in the DLSA group and 19 (range 8.0-27.0) with significance between groups.

\section{Discussion}

This study represents intrathecal administration of $8 \mathrm{mg}$ of dexamethasone with $12.5 \mathrm{mg}$ of $0.5 \%$ levobu- pivacaine received for surgical treatment of proximal femoral fractures, as an anaesthetic pattern which reduces the stress response by decreasing plasma cortisol levels with better quality analgesia and hospitalisation.

Enlightening the receptor types on neuronal presynaptic and postsynaptic membrane responsible for nociceptive signal transmission in dorsal roots of the spinal cord, intrathecal usage of low dose drugs was made possible as an effective method for perioperative pain management with lower incidence of unwanted events.

Intrathecal administration of glucocorticoids was presented during the $1980 \mathrm{~s}^{9}$. Administration of dexamethasone has the potential to inhibit patient's endogenous secretion of cortisol and when administered intrathecally, causes a short suppression of the pituitary-adrenal axis $^{10}$. Since then, intrathecal injections of steroids were frequently used for the treatment of mumps meningitis, chronic lymphocytic leukaemia and central nervous involvement in lupus erythematosus $^{11}$. Dexamethasone has an effectiveness of 36-54 hours and it is a long lasting corticosteroid ${ }^{12}$. This synthetic corticosteroid administered intrathecally affects nuclear transcription in adrenergic receptors. It inhibits corticosterone binding to type II of adrenergic receptors in the pituitary gland passing through the cerebrospinal fluid (CSF) bound to proteins. Irrelevantly of the concentration, dexamethasone has a similar effect to type II of adrenergic receptors. It is possible that it resorbs somewhere in the brain without an effect on other types of receptors along the HPA axis not depending on the concentration ${ }^{13}$. Intrathecal addition of dexamethasone to bupivacaine for elective orthopaedic surgery on lower limbs significantly prolongs duration of sensory block and decreases opioid requirements in postoperative management ${ }^{14}$. In several studies on peripheral nerve blocks when added to the local anaesthetic, dexamethasone prolongs sensor and motor blockade with significantly reduced postoperative analgesic requirements, which means it can inhibit phospholipase-A2 and cyclooxygenase- 2 expression during inflammation decreasing prostaglandin synthesis. Prolonged block may be the result of local effect on nerve fibers ${ }^{15,16}$. Single shot effect of intrathecal administration of dexamethasone with levobupivacaine in emergency patients with femoral fractures have not yet been evaluated in scientific literature. 
In various clinical studies on lowering perioperative stress effects, when spinal anaesthesia was used it has been proven to be most consistent block for surgical procedures from below the umbilicus region. The effectiveness of spinal anaesthesia results with lower immunosuppression via maintaining the number of T1 helper cells (Th1 cells) and by that stimulating cell immunity ${ }^{17}$. Inhibition of the natural killer cells (NKcells) function is related with the suppression of cortisol secretion ${ }^{18}$. When comparing regional and general anaesthesia, cortisol concentrations were reduced in the group that received spinal anaesthesia ${ }^{19}$. These results had shown that cortisol secretion induced by surgical stress is decreased in regional anaesthesia. Therefore, it is clear that afferent neural block induced by spinal anaesthesia decreases intraoperative and postoperative neuroendocrine stress response $\mathrm{e}^{20,21,22}$.

Delirium can refer to a cognitive complication prolonging hospitalisation and is associated with increased cortisol concentrations ${ }^{23}$. Postoperative period in elderly patients with proximal femoral fracture is characterized by a simultaneous elevation of plasma glucose and insulin concentrations, accompanied by a sustained high plasma cortisol concentration. It seems more probable that the prolonged changes seen in the elderly patients with fractures of the proximal femur reflect the effects of immobility after injury and operation, since it is known that physical inactivity leads to glucose intolerance ${ }^{24}$. The implications of these persisting high plasma concentrations of glucose, insulin and cortisol for the recovery of the patient with a proximal femoral fracture may be substantial. These findings therefore suggest that early mobilisation of elderly patients with proximal femoral fractures, in addition to other generally accepted benefits, might also prevent the development of deleterious metabolic abnormalities ${ }^{25}$.

Our study's correlation data of pain intensity and cortisol concentrations showed significant negative correlations between cortisol concentrations preoperatively and one hour after surgery with VAS scores measured in the same times reflecting longer hospitalisation in the LSA group pointing out that acute injury and perioperative stress effect had been reduced when dexamethasone was added intrathecally with enhancement of treatment outcome.

Patients in the study group had significantly lower pain intensity evaluated in VAS scores and without opioid analgesic requirements. Study results showed longer duration of analgesia in the study group, the DLSA group. Duration of analgesia was associated with the decreased cortisol concentrations.

Data showed the increase of serum glucose concentration one hour after surgery and on the tenth postoperative day which correlated with surgery duration in the LSA group, meaning the longer the duration of surgical procedure the more extended was the stress response in contrast to the DLSA group where there was not significance.

Duration of analgesia was significantly shorter in the control group according to the VAS scores measured with much increased opioid requiremnets postoperatively. Additionally cortisol concentrations measured in the LSA group were significantly higher, hospitalisation was longer and correlated with higher VAS scores. That reflected on shorter analgesia duration in comparison to the study group - the DLSA group - indicating the direct effect of dexamethasone with the local anaesthetic in CSF as significant beneficial adjuvant which relegates to spinal anaesthesia when only the local anaesthetic is administered.

Postraumatic stress disorder is an eminent risk factor for high morbidity and mortality rate with prolonged hospitalisation. From this study findings, sugical treatment of proximal femoral fracture in spinal anaesthesia presents shorter hospitalisation in the group that received dexamethasone DLSA/LSA $14.50 / 19.00$, which is also confirmed in the other studies, regional anaesthesia relates with better recovery and shorter length of hospital stay ${ }^{26}$.

\section{Conclusions}

Single shot of intrathecally administered dexamethasone with levobupivacaine received for surgical treatment of proximal femoral fractures reduces the stress response by decreasing plasma cortisol concentrations with longer lasting analgesic effect with better rehabilitation possibilities, hence shortening hospitalisation which explains this pattern of anaesthesia as first choice method.

\section{Acknowledgements}

The authors thank the Medical Staff, Division of Traumatology, Department of Surgery and Laborato- 
ry Division, University Hospital “Sveti Duh", Zagreb, School of Medicine, University of Zagreb for their support and assistance.

Conflicts of Interest: The authors declare no conflicts of interest.

\section{References}

1. Selye H. The stress of life. 1st ed. New York: McGraw-Hill; 1956.

2. Melzack R, Wall DP. Handbook of Pain Management. 1st ed. Churchill-Livingstone; 2003.

3. Chrousos GP, Gold PW. The concept of stress and stress system disorders. JAMA. 1992;267:1244-52.

4. Sapolsky RM. Why zebras don't get ulcers: a gude to stress, stress related diseases, and coping. 1st ed. New York:WH Freeman; 1994.

5. Bisschop PH, Klein S, Ackermans MT, Patterson BW, Endert E, van Lanschot JJ, et al. Pre-operative nutritional status does not alter the metabolic response to major gastrointestinal surgery in patients with oesophageal cancer. Br J Nutr. 2007; 98:181-6. https://doi.org/10.1017/S0007114507695567

6. Aubrun F. Management of postoperative analgesia in elderly patients. Reg Anesth Pain Med. 2005;30:363-79.

7. Ata A, Lee J, Bestle SL, Desemone J, Stain SC. Postoperative hyperglycemia and surgical site infection in general surgery patients. Arch Sur. 2010;145:858-64. https://doi.org/10.1001/ archsurg.2010.179

8. van Rijen EA, Harvey RA, Barton RN, Rose JG, Horan MA. Sensitivity of mononuclear leucocytes to glucocorticoids in elderly hip-fracture patients resistant to suppression of plasma cortisol by dexamethasone. Eur J Endocrinol. 1998;138: 659-66.

9. Chernow B, Vigersky R, O’Brien JT, Georges LP. Secondary adrenal insufficiency after intrathecal steroid administration. J Neurosurg. 1982;56:567-70.

10. Macro M, Reznik Y, Leymarie P, Loyau G, Mahoudeau J. The effect of Intrathecal dexamethasone injection on plasma cortisol level. Br J Rheumatol. 1991;30:238

11. Dong Y. Zhang X, Tang F, Tian X, Zhao Y, Zhang F. Intrathecal injection with methotrexate plus dexamethasone in the treatment of central nervous system involvement in systemic lupus erythematosus. Chin Med J. 2001;114:764-7.

12. O'Sullivan BT, Cutler DJ, Hunt GE, Walters C, Johnson GF, Caterson ID.Pharmacokinetics of dexamethasone and its relationship to dexamethasone suppression test outcome in depressed patients and healthy control subjects. Biol Psychiatry. 1997;41:574-84.

13. Miller AH, Spencer RL, Pulera M, Kang S, McEwen BS, Stein $\mathrm{M}$. Adrenal steroid receptor activation in rat brain and pituitary following dexamethasone: implications for the dexamethasone suppression test. Biol Psychiatry. 1992;32:850-69.
14. Bani-Hashem N, Hassan-Nasab B, Pour EA, Maleh PA, Nabavi A, Jabbari A. Addition of intrathecal dexamethasone to bupivacaine for spinal anaesthesia in orthopaedic surgery. Saudi J Anaesth. 2011;5:382-6. https://doi.org/10.4103/1658354X.87267

15. Yao XL, Cowan MJ, Gladwin MT, Lawrence MM, Angus $\mathrm{CW}$, Shelhamer JH. Dexamethasone alters arachidonate release from human epithelial cells by induction of $\mathrm{p} 11$ protein synthesis and inhibition of phospholipase A2 activity. J Biol Chem.1999;274:17202-8.

16. Movafegh A, Razazian M, Hajimaohamadi F, Meysamie A (2006) Dexamethasone added to lidocaine prolongs axillary brachial plexus blockade. Anesth Analg 102:263-267. https:// doi.org/10.1213/01.ane.0000189055.06729.0a

17. Le Cras AE, Galley HF, Webster NR. Spinal but not general anesthesia increases the ratio of $\mathrm{T}$ helper 1 to $\mathrm{T}$ helper 2 cell subsets in patients undergoing transurethral resection of the prostate. Anesth Analg. 1998;87:1421-5.

18. Brix-Christensen V, Tonnesen E, Sorensen IJ, Bilfinger TV, Sanchez RG, Stefano GB. Effects of anaesthesia based on high versus low doses of opioids on the cytokine and acute-phase protein responses in patients undergoing cardiac surgery. Acta Anaesth Scand. 1998;42:63-70.

19. Hogevold HE, Lyberg T, Kahler H, Haug E, Reikeras O. Changes in plasma IL-1beta, TNF-alpha and IL-6 after total hip replacement surgery in general or regional anaesthesia. Cytokine. 2000;12:1156-9. https://doi.org/10.1006/cyto.2000.0675

20. Kehlet H. [Surgery in the elderly]. Ugeskrift for laeger. 2000;162:641-3.

21. Wada H, Seki S, Takahashi T, Kawarabayashi N, Higuchi H, Habu Y, et al. Combined spinal and general anesthesia attenuates liver metastasis by preserving TH1/TH2 cytokine balance. Anesthesiology. 2007;106:499-506.

22. Liu S, Carpenter RL, Neal JM. Epidural anesthesia and analgesia. Their role in postoperative outcome. Anesthesiology. 1995;82:1474-506.

23. Bisschop PH, de Rooij SE, Zwinderman AH, van Oosten HE, van Munster BC. Cortisol, insulin, and glucose and the risk of delirium in older adults with hip fracture. J Am Geriatr Soc. 2011;59:1692-6. https://doi.org/10.1111/j.1532-5415.2011.03575.x

24. Frayn KN, Stoner HB, Barton RN, Heath DF, Galasko CS. Persistence of high plasma glucose, insulin and cortisol concentrations in elderly patients with proximal femoral fractures. Age Ageing. 1983;12:70-6.

25. Blotner H. Effect of prolonged physical inactivity on tolerance of sugar. Arch Int Med. 1945:75;39-44.

26. Neuman MD, Rosenbaum PR, Ludwig JM, Zubizarreta JR, Silber JH.Anesthesia technique, mortality and length of stay after hip fracture surgery. JAMA. 2014;311:2508-17. https:// doi.org/10.1001/jama.2014.6499 
Sažetak

\title{
DEKSAMETAZON- INTRATEKALNI REDUCENS JEDNOSTAVNIH HEMATOLOŠKIH BIOMARKERA STRESA
}

\begin{abstract}
L. Šakić, D. Tonković i K. Šakić
Proksimalni prijelomi bedrene kosti su najčešći uzrok prijma u bolnicu s visokim poslijeoperacijskim morbiditetom. Hipoteza je da jedna intratekalna doza deksametazona s levobupivakainom za kiruršku korekciju prijeloma proksimalnog dijela bedrene kosti smanjuje kirurški stres sa kvalitetnijim boravkom u bolnici. U istraživanje je bilo uključeno šezdeset pacijenata starije dobi s prijelomom proksimalnog dijela bedrene kosti, ASA status 2 i 3, te su bili randomizirani u dvije skupine. Trideset pacijenata je primilo $8 \mathrm{mg}$ deksametazona i $12,5 \mathrm{mg} \mathrm{0,5} \%$ levobupivakaina intratekalno, DLSA skupina,

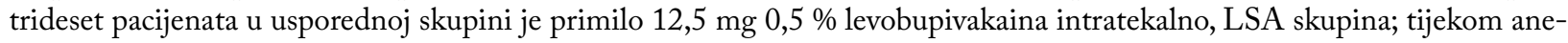
stezije za kiruršku korekciju. Plazmatske koncentracije kortizola i glukoze mjerene su prije i poslije kirurškog zahvata, intenzitet boli je određivan zbrojem VAS, te je analizirano trajanje hospitalizacije. Rezultati istraživanja su prikazali smanjene koncentracije kortizola, dulje trajanje analgezije te kraće trajanje hospitalizacije u DLSA skupini u usporedbi sa LSA skupinom. Koncentracije serumske glukoze nisu se značajnije razlikovale između skupina. Rasvjetljavanjem skupljenih rezultata, jedna intratekalna injekcija deksametazona u spinalnoj anesteziji za kiruršku korekciju prijeloma proksimalnog dijela bedrene kosti snižava stresni odgovor smanjenjem plazmatske koncentracije kortizola s duljim analgetskim učinkom te boljim mogućnostima rehabilitacije, skraćujući time hospitalizaciju što opravdava ovaj anesteziološki obrazac kao metodu izbora.
\end{abstract}

Ključne riječi: kortizol, glukoza, stres, bol, spinalna anestezija, prijelom bedrene kosti, hospitalizacija 